\title{
Optical images from Pathophysiological signals within breast tissue using three-dimensional near-infrared light
}

\author{
Hamid Dehghani $^{\dagger^{*}}$, Brian W Pogue ${ }^{\dagger}$, Shudong Jiang ${ }^{\dagger}$, Steven Poplack ${ }^{*}$ and Keith D Paulsen ${ }^{\dagger}$ \\ 'Thayer School of Engineering, Dartmouth College, Hanover, NH 03755 \\ *Dartmouth-Hitchcock Medical Center, Department of Radiology, Lebanon, NH 03766
}

\begin{abstract}
Near Infrared (NIR) tomography has the potential for characterization of different tissue types based upon cellular and vascular alternations that are optically apparent. This is especially useful for characterizing cancerous regions within normal tissue due to the high available contrast. Reconstructed images from NIR light propagation measurements through the female breast hold promise of providing clinically useful information about the pathophysiologic change of the tissue. We have developed a fast three-dimensional finite element model and image reconstruction algorithm, NIRFAST (Near Infrared Frequency-Domain Absorption and Scatter Tomography) and have previously tested the results extensively against simulated and phantom data[1]. The results have shown that the reconstructed images have good accuracy in recovering optical changes within the medium under investigation, and that with appropriate constraints, the calculated quantitative values agree well with the true values. Based on these algorithms, we present true three-dimensional images of the breast, from patient data. These images, which are reconstructed using NIR measurements over a range of wavelengths, provide additional information regarding the blood content and oxygen saturation distribution within the breast.
\end{abstract}

\section{INTRODUCTION}

Near Infrared optical tomography is a three-dimensional imaging method which can reconstruct physiologically-relevant chromophore distributions from the region under investigation [1-7]. Light is transmitted through tissue using multiple input and output locations on the surface of the region to be imaged, similarly to a fan-beam x-ray computed tomography geometry, but using optical fibers for delivery and pickup of the light signals. The intensity and pathlength distributions of the exiting photons provide information about the optical properties of the transilluminated tissue using a model-based interpretation where photon propagation is simulated by diffusion theory. Through iterative solution matching the theoretical prediction to the actual measured values, images of internal absorption and scattering coefficient distribution can be reconstructed. The main interest in this study lies in the ability to detect and characterize tumors within the female breast $[1,2]$. Since the absorption and scattering of light in tissue is a function of its optical properties, and hence its physiological state, our aim is to obtain images of internal optical absorption $\mu_{\mathrm{a}}$, reduced scattering $\mu_{\mathrm{s}}$ ', and ultimately images of total hemoglobin and oxygen saturation distributions. These images should in principle provide information about the physiological state of the tissue under investigation and help identify and characterize tumors within the breast.

The spatial resolution in NIR imaging is limited due to the high scattering of tissue under investigation, but NIR imaging does offer the evidence of providing high contrast imaging. Since light is attenuated similarly for both absorption and scattering heterogeneities, accurate quantitative imaging is a difficult task. Solutions to this problem come through the use of frequency-domain and time-resolved techniques to obtain pathlength information, in combination with modelbased iterative reconstruction methods that allow for the separation of absorption and scattering heterogeneities [1] and yield improvements in both spatial resolution and contrast [8].

The majority of modeling and image reconstruction studies have involved two-dimensional (2D) assumptions, yet a three-dimensional (3D) treatment of light propagation in tissue provides a more accurate prediction of the fluence distribution in the medium. Recently there has been significant progress in developing 3D modeling and image reconstruction 9-11], which is computationally more complex but also more accurate. As 3D image reconstruction becomes more developed it is crucial to define the resolution and contrast available from these algorithms

* email: Hamid.Dehghani@dartmouth.edu, tel (603) 646-9193, fax (603) 646-3856 
analogously to the studies which have been completed in 2D [12]. This is particularly true when using experimental data, since theoretical simulations will typically not represent the level of noise and systematic error present in actual data sets. Indeed, our studies have shown that it is the systematic errors which limit 2D diffusion tomography, and ultimately constrain the resolution and contrast which can be recovered [13-15]. Most studies have shown that even though images of optical absorption and scatter can be reconstructed simultaneously, the quantitative accuracy of these quantities remains relatively poor in the absence of special regularization schemes. Nonetheless, since absorption is a function of wavelength and blood concentration and therefore oxygen saturation, they can be used to produce maps of blood distribution and oxygen saturation of tissue under investigation.

In this work, we present true three-dimensional images of the breast, from patient data. These images, which are reconstructed using NIR measurements over a range of wavelengths, are used to provide additional information regarding the blood content and oxygen saturation distribution within the breast. These distributions can be used to help characterize and identify different type of cancerous tumors.

\section{THE FORWARD PROBLEM}

In our problem, we solve the frequency domain version of this equation:

$$
-\nabla \cdot D \nabla \Phi(\mathbf{r}, \omega)+\left(\mu_{a}+\frac{i \omega}{c}\right) \Phi(\mathbf{r}, \omega)=S_{0}(\mathbf{r}, \omega)
$$

where $\omega$ is the intensity-modulation frequency of the light signal. This latter version is solved in discrete form using the finite element method. The fluence is a complex field (i.e. it has both a real and imaginary component), which is in turn mapped to amplitude and phase.

The best description of the air-tissue boundary is derived with an index-mismatched Robin-type condition, where the fluence at the edge of the tissue exits and does not return. Thus the flux leaving the boundary is equal to the fluence rate at the boundary, times a factor which accounts for the internal reflection of light back into the tissue. This relationship is described in the following equation $([16,17])$ :

$$
\Phi(\mathbf{r}, \omega)=2 A n \cdot D \nabla \Phi(\mathbf{r}, \omega)
$$

The value of A depends upon the relative refractive index mismatch between tissue and air. We implement the finite element method (FEM) to model the diffusion equation, which is attractive due to the flexibility in terms of geometry and the ability to preserve the non-linear relationship between the measurements and the properties in Equation (1).

\section{THE INVERSE PROBLEM}

In the inverse problem (image reconstruction), the goal is recovery of $\mu_{a}$ and $\mu_{s}^{\prime}$ at each FEM node based on measurements of optical flux at the tissue surface. Most image reconstruction algorithms are achieved numerically by minimizing an objective function, which depends on the difference between measured data, $\Phi^{M}$, and calculated data, $\Phi^{C}$, from the forward solver. Here, $\Phi^{C}$, implicitly depends upon position, frequency and the spatial distribution of $\mu_{a}$ and $\mu_{s}^{\prime}$. Typically this is written as the minimization of $\chi^{2}$ :

$$
\chi^{2}=\sum_{i=1}^{N M}\left(\Phi_{i}^{C}-\Phi_{i}^{M}\right)^{2}
$$


where $i$ is the index for each source detector pair, and NM is the total number of measurements. $\chi^{2}$ can be minimized in a least squares sense, setting the derivative equal to zero, and using a Newton-Raphson approach. We use a Levenberg Marquardt algorithm, to repeatedly solve the equation:

$$
a=\left(J^{T} J+\lambda I\right)^{-1} J^{T} b
$$

where $b$ is the data vector, $b=\left(\Phi^{C}-\Phi^{M}\right)^{T} ; a$ is the solution update vector, $a=\left\lfloor\delta D_{j} ; \delta \mu_{a j}\right\rfloor$, defining the difference between the true and estimated optical properties at each reconstructed node $j$. Here, $\lambda$ is a regularization factor to stabilize matrix inversion and $J$ is the Jacobian matrix for our model, which is calculated using the Adjoint method[18]. It has the form:

$$
J=\left[\begin{array}{cccccccc}
\frac{\delta \ln I_{1}}{\delta D_{1}} & \frac{\delta \ln I_{1}}{\delta D_{2}} & \cdots & \frac{\delta \ln I_{1}}{\delta D_{j}} ; & \frac{\delta \ln I_{1}}{\delta \mu_{a 1}} & \frac{\delta \ln I_{1}}{\delta \mu_{a 2}} & \cdots & \frac{\delta \ln I_{1}}{\delta \mu_{a j}} \\
\frac{\delta \theta_{1}}{\delta D_{1}} & \frac{\delta \theta_{1}}{\delta D_{2}} & \cdots & \frac{\delta \theta_{1}}{\delta D_{j}} ; & \frac{\delta \theta_{1}}{\delta \mu_{a 1}} & \frac{\delta \theta_{1}}{\delta \mu_{a 2}} & \cdots & \frac{\delta \theta_{1}}{\delta \mu_{a j}} \\
\frac{\delta \ln I_{2}}{\delta D_{1}} & \frac{\delta \ln I_{2}}{\delta D_{2}} & \cdots & \frac{\delta \ln I_{2}}{\delta D_{j}} ; & \frac{\delta \ln I_{2}}{\delta \mu_{a 1}} & \frac{\delta \ln I_{2}}{\delta \mu_{a 2}} & \cdots & \frac{\delta \ln I_{2}}{\delta \mu_{a j}} \\
\frac{\delta \theta_{2}}{\delta D_{1}} & \frac{\delta \theta_{2}}{\delta D_{2}} & \cdots & \frac{\delta \theta_{2}}{\delta D_{j}} ; & \frac{\delta \theta_{2}}{\delta \mu_{a 1}} & \frac{\delta \theta_{2}}{\delta \mu_{a 2}} & \cdots & \frac{\delta \theta_{2}}{\delta \mu_{a j}} \\
\vdots & \vdots & \ddots & \vdots & \vdots & \vdots & \ddots & \vdots \\
\frac{\delta \ln I_{S}}{\delta D_{1}} & \frac{\delta \ln I_{S}}{\delta D_{2}} & \cdots & \frac{\delta \ln I_{S}}{\delta D_{j}} ; & \frac{\delta \ln I_{S}}{\delta \mu_{a 1}} & \frac{\delta \ln I_{S}}{\delta \mu_{a 2}} & \cdots & \frac{\delta \ln I_{S}}{\delta \mu_{a j}} \\
\frac{\delta \theta_{S}}{\delta D_{1}} & \frac{\delta \theta_{S}}{\delta D_{2}} & \cdots & \frac{\delta \theta_{S}}{\delta D_{j}} ; & \frac{\delta \theta_{S}}{\delta \mu_{a 1}} & \frac{\delta \theta_{S}}{\delta \mu_{a 2}} & \cdots & \frac{\delta \theta_{S}}{\delta \mu_{a j}}
\end{array}\right]
$$

where $\frac{\delta \ln I_{i}}{\delta D_{j}}$ and $\frac{\delta \ln I_{i}}{\delta \mu_{a j}}$ are the sub-matrices that define the relation between the log of the amplitude of the $i^{\text {th }}$ measurement with respect to $D$ and $\mu_{a}$ at the $j^{\text {th }}$ reconstructed nodes respectively; $\frac{\delta \theta_{i}}{\delta D_{j}}$ and $\frac{\delta \theta_{i}}{\delta \mu_{a j}}$ are the submatrices that define the relation between the phase of the $i^{\text {th }}$ measurement with respect to $D$ and $\mu_{a}$ at the $j^{\text {th }}$ node respectively. $S$ is the total number of measurements.

Finally in the inverse problem, where we aim to recover internal optical property distributions from boundary measurements, we assume that $\mu_{\mathrm{a}}(\mathbf{r})$ and $\mu_{\mathrm{s}}^{\prime}(\mathbf{r})$ are expressed in a basis with a limited number of dimensions (less than the dimension of the finite element system matrices). A number of different strategies for defining reconstruction bases are possible; in this paper we use a second mesh basis [19], whose local shape and continuity characteristics are the same as the original mesh, but with fewer degrees of freedom.

\section{METHOD AND RESULTS}

In the following section, we describe and present reconstructed images of internal absorption and scatter from 2 sets of patient data collected at multiple wavelengths, ranging from $761 \mathrm{~nm}$ to $826 \mathrm{~nm}$. For the NIR exam the attendant brought the fiber optic imaging array into direct contact with the breast at the level of the clinical abnormality. We obtained three tomographic acquisitions centered at the tumor site, with contiguous slices above and below the primary plane of interest, as indicated in Fig. 1. Measurements collected from a cylindrical phantom at each wavelength were also recorded for calibration purposes. 
From knowledge of the diameter of each measurement plane and of the separation between planes, we constructed a conical shaped mesh. See figure 2(a) for an example. For image reconstruction, each data set was calibrated for each wavelength according to the procedures described elsewhere [1]. For the reconstruction basis a second mesh of the same geometry was used. See figure 2(b) for an example. The reconstruction time after the initial calibration procedure was approximately 10 min per iteration on a $1.7-\mathrm{GHz}$ PC with 2 Gigs of RAM.

We describe the clinical and NIR imaging of the first subject (volunteer A). The patient presented for standard screening mammography, which revealed a subtle nodular density and associated architectural distortion in the lateral aspect of the right breast. Pathology showed an invasive carcinoma of $20 \mathrm{~mm}$ size. The patient was presented for NIR measurement a soon after biopsy.

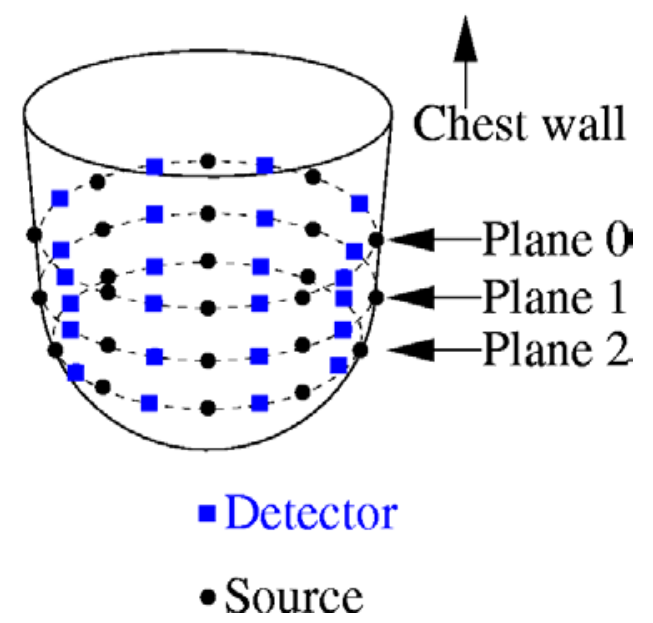

Figure 1. A diagram of the setup used for the data collection from the patients is shown. Measurements were made one plane at a time, with the mid-plane positioned at the midline of the lesion. In each case the radius of the measurement plane was recorded, as well the separation between each plane. For mesh generation, $20 \mathrm{~mm}$ was allowed below and above the top and bottom planes of the fiber optic array.

(a)

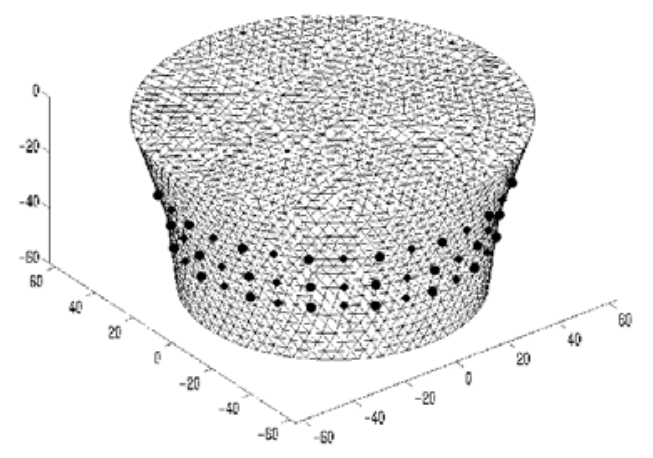

Figure 2. Example of a mesh used for the reconstruction of images from measured clinical data. The information about data collection geometry that was used for mesh generation included slice diameter, allowing generation of: (a) a conical shaped mesh used for the calculation of the Jacobian; (b) a conical shaped mesh used for the reconstruction basis. 
Three-dimensional images of internal absorption and reduced scatter were reconstructed simultaneously from NIR data collected at each wavelength. The mesh used for the calculation of the Jacobian contained 8334 nodes corresponding to 41623 linear tetrahedral elements. For reconstruction basis, a second mesh of the same geometry was used, but with 2521 nodes, corresponding to 11575 linear tetrahedral elements. Images were reconstructed at 4 wavelengths of 761 , 785,808 and $826 \mathrm{~nm}$, and they are shown in Figure 3. For image reconstruction the regularization parameter $(\lambda)$ used was initially set to 10 , and was allowed to decrease by a factor of $10^{1 / 4}$ if the projection error $\left(\chi^{2}\right.$, equation 3$)$ had decreased with respect to previous iteration. Images shown are those at the $10^{\text {th }}$ iteration. Here the images are true three dimensional reconstructions and coronal slices at $\mathrm{z}=-60,-45,-30,-15$ and $0 \mathrm{~mm}$ are only shown. From the reconstructed images it can be seen that an anomaly is found within the mid-plane at approximately 9 o'clock position. The anomaly is showing an absorption variation with wavelength, whereas the reduced scatter is almost constant over all reconstructed wavelengths. The absorption images were used together with published values of extinction coefficients for oxy and deoxy hemoglobin [20] $\left(\mathrm{HbO}_{2}\right.$ and $\mathrm{Hb}$ respectively) for the calculation of $\mathrm{Hb}, \mathrm{HbO}_{2}$, total hemoglobin $(\mathrm{HbT})$ and oxygen saturation $\left(\mathrm{SO}_{2}\right)$. The calculated three dimensional maps of $\mathrm{Hb}, \mathrm{HbO}_{2}$, total $\mathrm{HbT}$ and $\mathrm{SO}_{2}$ are also shown in figure 3.

S61 nm

Figure 3. Reconstructed images of absorption (top row) and reduced scatter (middle row) at each wavelength from measured volunteer data, (volunteer A), as well as calculated maps of blood content; each slice represents a plane through the mesh, from the bottom near the nipple to the top near the chest. The images are coronal views of the cross section through the breast at the tenth iteration at the wavelengths indicated.

From the calculated values of $\mathrm{Hb}$ it is seen that the anomaly is showing a peak value of $47.7 \mu$ Mole, compared to a background of $32.87 \mu$ Mole, whereas the $\mathrm{HbO} 2$ image is showing a peak value of $24.73 \mu$ Mole at a location on the 
periphery of the skin. The total hemoglobin value is also showing a peak at the location of the anomaly, with a value of $65.28 \mu$ Mole. SO2 values, calculated by taking the ratio of oxygenated blood and total blood content, is showing a marked decrease at the location of the anomaly, with a value of $25.9 \%$, as compared to a background value of $38.11 \%$. These data are summarized in Table 1.

A second patient volunteer was presented for standard screening mammography, which after biopsy revealed a benign ductal hyperplasia region within the right breast. As in the previous case, data at multiple wavelengths were measured and used for calculation of internal optical properties. The mesh used for the calculation of the Jacobian contained 9259 nodes corresponding to 45613 linear tetrahedral elements. For reconstruction basis, a second mesh of the same geometry was used, but with 1916 nodes, corresponding to 8140 linear tetrahedral elements. Images were again reconstructed at 4 wavelengths of 761, 785, 808 and $826 \mathrm{~nm}$, and they are shown in Figure 4. For image reconstruction the regularization parameter $(\lambda)$ used was initially set to 10 , and was allowed to decrease by a factor of $10^{1 / 4}$ if the projection error $\left(\chi^{2}\right.$, equation 3) had decreased with respect to previous iteration. Images shown are those at the $10^{\text {th }}$ iteration. Here as before, the images are true three dimensional reconstructions and coronal slices at $\mathrm{z}=-60,-45,-30,-15$ and $0 \mathrm{~mm}$ are only shown. From the reconstructed images it can be seen that an anomaly is found within the mid-plane at approximately off center near the 9 o'clock position.

(761 nm

Figure 4. Reconstructed images of absorption (top row) and reduced scatter (middle row) at each wavelength from measured volunteer data, (volunteer B), as well as calculated maps of blood content; each slice represents a plane through the mesh, from the bottom near the nipple to the top near the chest. The images are coronal views of the cross section through the breast at the tenth iteration at the wavelengths indicated. 
Here, the anomaly is showing variation of absorption with wavelength, with a peak value of $0.0055 \mathrm{~mm}^{-1}$ at $761 \mathrm{~nm}$, and the reduced scatter images are showing a peak value of $3.3 \mathrm{~mm}^{-1}$, also at $761 \mathrm{~nm}$. The calculated maps of $\mathrm{Hb}$ is showing a value of $10.76 \mu$ Moles at the location of the anomaly, compared to a background of mean value $7.7 \mu$ Moles. The $\mathrm{HbO}_{2}$ image is showing a max value of $15.56 \mu$ Moles, compared to a mean background value of $8.4 \mu$ Moles. From the SO2 images, is can be seen that the anomaly is showing a marked increase of $69.26 \%$ saturation, compared to a mean background saturation of $51.52 \%$. These data are summarized in Table 1.

\begin{tabular}{c|ccccc} 
& $\begin{array}{c}\mathrm{Hb} \\
(\mu \mathrm{Moles})\end{array}$ & $\mathrm{HbO}_{2}(\mu$ Moles $)$ & $\mathrm{HbT}(\mu$ Moles $)$ & $\mathrm{SO}_{2}(\%)$ & Tumor Type \\
\hline $\begin{array}{c}\text { Volunteer A } \\
\text { (anomaly) }\end{array}$ & $47.7 \uparrow$ & $24.73 \uparrow$ & $65.28 \uparrow$ & $25.9 \downarrow$ & $\begin{array}{c}\text { Invasive ductal } \\
\text { carcinoma }\end{array}$ \\
$\begin{array}{c}\text { Volunteer A } \\
\text { (background) }\end{array}$ & 32.87 & 20.23 & 53.11 & 38.11 & \\
$\begin{array}{c}\text { Volunteer B } \\
\text { (anomaly) }\end{array}$ & $10.76 \uparrow$ & $15.56 \uparrow$ & $22.56 \uparrow$ & $69.26 \uparrow$ & $\begin{array}{c}\text { Benign ductal } \\
\text { hyperplasia }\end{array}$ \\
$\begin{array}{c}\text { Volunteer B } \\
\text { (background) }\end{array}$ & 7.71 & 8.40 & 16.11 & 51.52 &
\end{tabular}

Table 1. Summarized values of Hemoglobin and oxygen saturation maps shown in Figures 3 and 4 . The arrows show the increase $(\boldsymbol{\uparrow})$ or decrease $(\downarrow)$ of values within the region of interest with respect to the background.

\section{DISCUSSIONS AND CONCLUSIONS}

In this work we have described and presented clinical NIR images measured from volunteers who had presented with anomalies within their breast. In each case, NIR data were measured at 4 wavelengths, ranging from $761 \mathrm{~nm}$ to $829 \mathrm{~nm}$, and three dimensional images of internal absorption and reduced scatter at each wavelength were reconstructed simultaneously from these data.

From volunteer A, who had shown a invasive ductal carcinoma within the breast, absorption and scatter images reconstructed showed an increase within the region of interest. The calculated values of absorption were used together with extinction coefficients of $\mathrm{Hb}, \mathrm{HbO}_{2}$ to calculate deoxy, oxy and total hemoglobin maps, and from these oxygen saturation images were calculated. While the total hemoglobin levels within the region of interest shows an increase with respect to background, the oxygen saturation shows a marked decrease within the same region. This trend is perhaps expected for a malignant tissue, as one would expect a rise in blood content, due to an increase in blood vessel density, but since malignant tumors are more active, this would result to a decrease in oxygen saturation.

From volunteer B, who had shown a benign ductal hyperplasia within the breast, absorption and scatter images reconstructed also showed an increase within the region of interest. The calculated values of absorption were again used together with extinction coefficients of $\mathrm{Hb}, \mathrm{HbO}_{2}$ to calculate deoxy, oxy and total hemoglobin maps, and from these oxygen saturation images were calculated. Here the total hemoglobin levels within the region of interest showed an increase with respect to background, as well as an increase of the oxygen saturation within the same region. This is again perhaps expected for a benign tissue, as one would expect a rise in blood content, due to an increase in blood vessel density, but since benign tumors are not particularly more active, this would result to an increase in oxygen saturation.

It should be stressed that these results are preliminary, and further investigation is needed before one can claim tumor classification from tissue oxygenation maps. Further work is in progress with respect to building a larger database of such images, whereby allowing a proper and definite statistical analysis of the results.

\section{ACKNOWLEDGEMENTS}

Funding for this work has been provided from National Cancer Institute through research grants PO1CA80139 and RO1CA69544. 


\section{REFERENCES}

1. Dehghani, H., Pogue, B. W., Poplack, S. P., Paulsen, K. D., Multiwavelength Three-Dimensional Near-Infrared Tomography of the Breast: Initial Simulation, Phantom, and Clinical Results. Applied Optics, 2003. 42(1): p. 135-145.

2. $\quad$ Pogue, B.W., Geimer, S., McBride, T. O., Jiang, S., Österberg, U. L., Paulsen, K. D., Three-dimentionsal Simulation of Near-Infrared Diffusion in Tissue: Boundary Condition and Geometry Analysis For Finite Element Image Reconstruction. Appl. Opt., 2001. 40(4).

3. Mcbride, T.O., Pogue B W , S. Jiang, U. L. Osterberg, K. D. Paulsen, and S. P. Poplack, Multi-spectral nearinfrared tomography: a case study in compensating for water and lipid content in hemoglobin imaging of the breast. J. Biomed. Opt., 2002. 7.

4. Hebden, J.C., H. Veenstra, H, H. Dehghani, E. M. C. Hillman, M. Schweiger, S. R. Arridge, and D. T. Delpy, Three dimensional time-resolved optical tomography of a conical breast phantom. Applied Optics, 2001. 40: $\mathrm{p}$. 3278-3287.

5. Fantini, S., M. A. Franceschini, E. Gratton, D. Hueber, W. Rosenfeld, D. Maulik, P. G. Stubblefield and M. R. Stankovic, Non-invasive optical mapping of the piglet in real time. Opt. Exp., 1999. 4: p. 308-314.

6. Eda, H., I. Oda, Y. Ito, Y. Wada, Y. Oikawa, Y. Tsunazawa, Y. Tsuchiya, Y. Yamashita, M. Oda, A. Sassaroli, Y. Yamada and M. Tamaru, Multichannel time-resolved optical tomographic imaging system. Rev. Sci. Instrum., 1999. 70: p. 3595-3602.

7. Boas, D.A., D. H. Brooks, E. L. Miller, C. A. DiMarzio, M. Kilmer, R. J. Gaudette, and Q. Zhang, Imaging the body with diffuse optical tomography. IEEE Signal Processing Magazine, 2001. 18(6): p. 57-75.

8. Jiang, H.B., et al., Frequency-domain optical image reconstruction in turbid media: An experimental study of single-target detectability. Applied Optics, 1997. 36(1): p. 52-63.

9. Schweiger, M. and S.R. Arridge, Comparison of two- and three-dimensional reconstruction methods in optical tomography. Applied Optics, 1998. 37(31): p. 7419-7428.

10. Jiang, H., Y, Xu, N. Iftimia, J. Eggert, K. Klove, L. Baron, and L. Fajardo, Three-dimensional optical tomographic imaging of breast in a human subject. IEEE Trans. Med. Img., 2001. 20(12): p. 1334-40.

11. Gibson, A., R. M. Yusof, E. M. C. Hillman, H. Dehghani, J. Riley, N. Everdale, R. Richards, J. C. Hebden, M. Schweiger, S. R. Arridge, and D. T. Delpy, Optical tomography of a realistic neonatal head phantom. Applied Optics, 2002. Accepted.

12. Pogue, B.W., et al., Contrast-detail analysis for detection and characterization with near-infrared diffuse tomography. Medical Physics, 2000. 27(12): p. 2693-2700.

13. Pogue, B.W., X. Song, T. D Tosteson, T. O. McBride, S. Jiang, and K. D. Paulsen, Stastical analysis of nonliearlly reconstructed near-infrared tomographic images: Part I-Theory and Simulation. IEEE Trans. Med. Img., 2002. 7(12): p. 755-763.

14. Song, X., Pogue B W, Tosteson TD, McBride T O, Jiang S, and Paulsen K D, Statistical Analysis of Nonlinearly Reconstructed Near-Infrared Tomographic Images: Part II - Experimental Interpretation. IEEE Trans. Med. Img., 2002. 7(12): p. 764 -772.

15. Dehghani, H., Pogue B W, Jiang, S., Brooksby, B., and Paulsen K D, Three Dimensional Optical Tomography: Resolution in Small Object Imaging. Applied Optics, 2003. Accepted.

16. Groenhuis, R.A.J., Ferwerda, H. A., ten Bosch, J. J., Scattering and absorption of turbid materials determined from reflection measurements. I.Theory. Appl. Opt., 1983. 22(16): p. 2456-2462.

17. Schweiger, M., S R Arridge, M Hiroaka and D T Delpy, The Finite Element Model for the Propagation of Light in Scattering Media: Boundary and Source Conditions. Med. Phys., 1995. 22: p. 1779-1792.

18. Arridge, S.R., and Schweiger, M., Photon-measurement density functions. Part2: Finite-element-method calculations. Applied Optics, 1995. 34: p. 8026-8037.

19. Paulsen, K.D., and Jiang H., Spatially varying optical property reconstruction using a finite element diffusion equation approximation. Med. Phys., 1995. 22(6): p. 691-701.

20. Wray, S., Cope, M., Delpy, D. T., Wyatt, J. S., Reynolds, E., Charaterization of the near infrared absorption spectra of cytochrome aa 3 and haemoglobin for the non-invasive monitoring of cerebral oxygenation.

Biochem. Biophys. Acta, 1988. 933: p. 184-192. 